\begin{tabular}{|c|l|}
\hline Title & Kinetics of nucleation in a dilute polymer solution \\
\hline Author(s) & Nakata, Mitsuo; Kawate, Kohichiro \\
\hline Citation & $\begin{array}{l}\text { PHY SICAL REVIEW LETTERS, 68, 2176-2179 } \\
\text { https://doi.org/10.1103/PhysRevLett.68.2176 }\end{array}$ \\
\hline Issue Date & 1992 \\
\hline Doc URL & http://hdl.handle.net/2115/5861 \\
\hline Rights & Copyright $\odot 1992$ A merican Physical Society \\
\hline Type & article \\
\hline File Information & PRL68-14.pdf \\
\hline
\end{tabular}

Instructions for use 


\title{
Kinetics of Nucleation in a Dilute Polymer Solution
}

\author{
Mitsuo Nakata and Kohichiro Kawate \\ Department of Polymer Science, Faculty of Science, Hokkaido University, Sapporo 060, Japan
}

(Received 10 December 1990)

To determine the time evolution of the droplet size distribution in the nucleation process, we have made light-scattering measurements on supercooled dilute solutions of poly(methyl methacrylate) in 1chlorobutane, which showed extremely slow phase separation. The observed scattered intensities were analyzed by the Guinier plot and represented in a scaled form. The time evolution of the mean radius $\bar{R}(t)$, total density $N(t)$, and droplet size distribution $N(R, t)$ of droplets of minority phase was given by the relations $\bar{R}(t) \sim t, N(t) \sim t^{0.6}$, and $N(R, t)=N(t) h(R / \bar{R}) / \bar{R}$, independent of quench depth and concentration.

PACS numbers: $64.60 . \mathrm{Qb}, 61.25 . \mathrm{Hq}$

By visual observation on dilute solutions of poly (methyl methacrylate) (PMMA) with high molecular weight in 1-chlorobutane $(\mathrm{BuCl})$, we found that the transparency of the supercooled solutions decreased gradually and sudden clouding marked as a cloud point was not observed. In some conditions the decrease of the transparency seemed to level off at a certain degree of clouding. Taking advantage of the above peculiar behavior in the phase-separation process, we have carried out lightscattering measurements on the dilute solutions of PMMA to determine the time evolution of the droplet size distribution of minority phase in the nucleation process.

Nucleation experiments on binary liquid mixtures have been performed by cloud-point measurements [1-4] and by using a microscope $[5,6]$. Analyses of droplet size data based on a recent nucleation theory [5,7] suggested that the data were obtained at a later stage of nucleation. In this study the mean radius $\bar{R}(t)$ and total density $N(t)$ of droplets as a function of time $t$ were represented by $\bar{R}(t) \sim t$ and $N(t) \sim t^{0.6}$ at small $t$. The increase of $N(t)$ with increasing $t$ indicates a nucleation process at which droplet birth is predominant. The time evolution of observed scattering curves and droplet size distribution was found to obey a scaling relation as observed in the case of a spinodal decomposition process $[8,9]$.

The PMMA sample used has been characterized by $M_{w^{\prime}}=2.44 \times 10^{6}$ and $M_{w} / M_{n}=1.20[10]$, where $M_{w}$ and $M_{n}$ are the weight- and number-average molecular weight, respectively [11]. $\mathrm{BuCl}$ was purified as described elsewhere [10]. We have employed two dilute solutions of the PMMA in $\mathrm{BuCl}$ with the concentration $C$ $=1.45 \times 10^{-3} \mathrm{~g} / \mathrm{ml}$ (solution $B$ ) and $2.91 \times 10^{-3}$ (solution $E$ ).

Light-scattering measurements were made with a HeNe laser $(632.8 \mathrm{~nm})$ as a light source. A large scattering cell of $14.0 \mathrm{~mm}$ i.d. was used to carry out the measurements in the angular range from $14^{\circ}$ to $40^{\circ}$ or $90^{\circ}$ and to detect weak scattering from droplets at the beginning of phase separation. The scattering cell was immersed in a silicone oil bath controlled to within $0.01 \mathrm{~K}$.

To obtain the coexistence curve the phase-separation temperature was measured by lowering the temperature very slowly. For dilute solutions the phase-separation temperature was determined as the temperature at which forward-scattering intensity began to increase and for concentrated solutions as the temperature at which incident-beam intensity after passing through the solution began to decrease by strong scattering. The preparation of uniform solutions for $C>0.07 \mathrm{~g} / \mathrm{ml}$ could hardly be made because of extremely high viscosity. For solutions $B$ and $E$ the phase-separation temperature was determined as 31.86 and $33.25^{\circ} \mathrm{C}$, respectively, by lowering the temperature about $0.02 \mathrm{~K} / \mathrm{h}$ stepwise. The observed coexistence curve is given in Fig. 1. The top of the curve gives the critical point as $T_{c}=36.1^{\circ} \mathrm{C}$ and $C_{c}=0.034$ $\mathrm{g} / \mathrm{ml}$. The time evolution of the scattering curves was measured from the time when the scattering cell, which had been kept $0.1 \mathrm{~K}$ above the phase-separation temperature, was immersed in the silicone oil bath. The measurement was performed at the quench depths $\delta T=0.10$, 0.30 , and $0.50 \mathrm{~K}$ for solution $B$ and at $\delta T=0.10 \mathrm{~K}$ for solution $E$, where $\delta T$ is measured from the coexistence curve as indicated in Fig. 1. The time delay due to thermal equilibrium attainment and incubation of nuclei was not usually noticeable. To correct for multiple scattering [12], the scattered intensity obtained relative

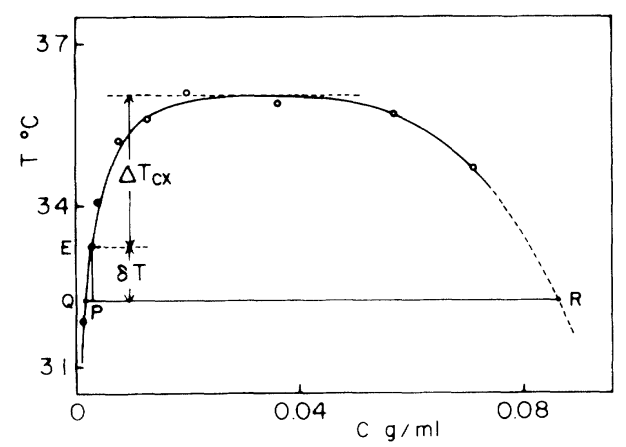

FIG. 1. Coexistence curve of PMMA in $\mathrm{BuCl}$ obtained with observed phase-separation temperatures. Point $E$ corresponds to solution $E$, which separates into two phases $Q$ and $R$ when quenched to $P$. Actual quench depth in scattering experiments was $\delta T=0.10 \mathrm{~K}$ for the solution. For off-critical binary mixtures the cloud point is usually located near $\delta T / \Delta T_{C}=0.2$ [3]. 
to the incident one was divided by the transmittance $F$ of the solution, which was higher than 0.5 in the present study. Scattered intensity from droplets of minority phase was determined as the difference between the corrected intensity and the background intensity measured at $0.1 \mathrm{~K}$ above the phase-separation temperature.

For a system of spherical particles having a size distribution such that $N(R) d R$ is the density of particles with radius in the range $R$ to $R+d R$, the theoretical scattered intensity is given by

$$
I(k)=A \int_{0}^{\infty} N(R)\left(R^{3} / k^{3}\right) J_{3 / 2}^{2}(k R) d R,
$$

where $A$ is related to the optical constant of the system, $k$ is the scattering vector $[(4 \pi / \lambda) \sin (\theta / 2)]$ in the medium, and $J_{3 / 2}(k R)$ is the Bessel function of order $\frac{3}{2}$. Riseman has solved Eq. (1) for $N(R)$ as [13]

$$
\begin{aligned}
& N(R)=\sqrt{\pi} /\left(2 A R^{3}\right) \int_{0}^{\infty} k^{3} I(k) \Phi(k R) d k \\
& \Phi(z)=(8 / \sqrt{z})\left[\left(2-z^{2}\right) J_{3 / 2}(2 z)-(3 / 2) z J_{1 / 2}(2 z)\right] .
\end{aligned}
$$

At small $k$ Eq. (1) could be approximated for practical purposes as [14]

$$
I(k)=I_{0} \exp \left(-0.221 \bar{R}^{2} k^{2}\right)
$$

where $I_{0}$ is the scattered intensity at zero angle and $\bar{R}$ is a mean radius of the particles.

In Fig. 2 observed time evolution of scattered intensity is depicted by the Guinier plot of $\log I(k, t)$ vs $k^{2}$ for solution $B$ at $\delta T=0.10 \mathrm{~K}$. Curves $a, b, c$, and $d$, which were measured at time $t=240$ (1.00), 270 (1.00), 330 (0.99), and $390 \mathrm{~min}(0.98)$, respectively, demonstrate typical behavior of observed scattering curves. Here, the values in the parentheses indicate the transmittance $F$ of the solution. Using Eq. (3) the mean radius $\bar{R}(t)$ and the zeroangle scattering intensity $I(0, t)$ were determined with a least-squares method from the data points at lower angles. Being disturbed by stray light we could not determine the radius of droplets smaller than $89 \mathrm{~nm}$, which is about twice as large as the unperturbed radius $40 \mathrm{~nm}$ of the polymer coil [10].

To see the power laws $\bar{R}(t)=a t^{\alpha}, I(0, t)=b t^{\beta}$, and $I(0, t)=c \bar{R}^{\gamma}$, we made the corresponding double logarith-

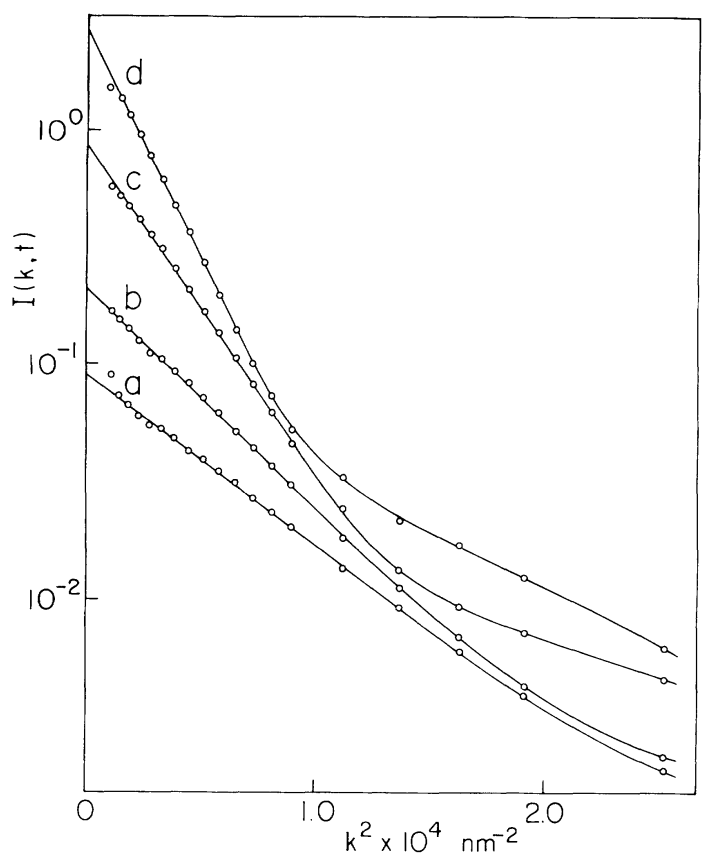

FIG. 2. Time evolution of the scattering curve by Guinier plot at quench depth $0.10 \mathrm{~K}$ for solution $B$. Curves $a, b, c$, and $d$ were obtained $240,270,330$, and $390 \mathrm{~min}$ after quench, respectively.

mic plots for the data of $I(0, t)$ and $\bar{R}(t)$. These plots were found to be represented by a straight line at small $t$ and the values of the exponents and standard deviations were determined by a least-squares method as given in Table I. Since $\alpha$ was close to unity, we made the plot of $\bar{R}(t)$ vs $t$ in Fig. 3. Curves $a, b$, and $c$ were for solution $B$ at the quench depths $\delta T=0.10,0.30$, and $0.50 \mathrm{~K}$, respectively, and curve $d$ is for solution $E$ at $\delta T=0.10 \mathrm{~K}$. The upright and upset triangles for curve $b$ show two different experimental runs carried out for the same solution. This is also the case for the open circles with and without bars for curve $d$. The arrows indicate the time at which the transmittance of the solution decreases to 0.90 . After that time the data of $\bar{R}(t)$ and $I(0, t)$ could be just apparent ones because of the approximate correction for the

\begin{tabular}{|c|c|c|c|c|}
\hline$\delta T(\mathrm{~K})$ & 0.10 & $\begin{array}{c}B \\
0.30\end{array}$ & 0.50 & $\begin{array}{c}E \\
0.10\end{array}$ \\
\hline$\alpha$ & $1.02 \pm 0.01$ & $0.92 \pm 0.03$ & $0.78 \pm 0.01$ & $0.99 \pm 0.02$ \\
\hline$\beta$ & $6.6 \pm 0.1$ & $5.9 \pm 0.1$ & $5.2 \pm 0.1$ & $6.5 \pm 0.1$ \\
\hline$\gamma$ & $6.5 \pm 0.1$ & $6.4 \pm 0.2$ & $6.7 \pm 0.1$ & $6.6 \pm 0.1$ \\
\hline$a(\mathrm{~nm} / \mathrm{min})$ & $1.15 \pm 0.01$ & $1.60 \pm 0.02$ & $2.25 \pm 0.07$ & $2.56 \pm 0.01$ \\
\hline$c \times 10^{18}$ & $8.3 \pm 0.3$ & $60 \pm 3$ & $123 \pm 2$ & $5.6 \pm 0.1$ \\
\hline$c a^{0.6} \times 10^{18}$ & 9 & 80 & 200 & 10 \\
\hline
\end{tabular}
multiple scattering.

TABLE I. Parameters for the relations $\bar{R}(t)=a t^{\alpha}, I(0, t) \sim t^{\beta}$, and $I(0, t)=c \bar{R}^{\gamma}(t)$. $a$ and $c$ were determined for the fixed value $\alpha=1$ and $\gamma=6.6$, respectively. 


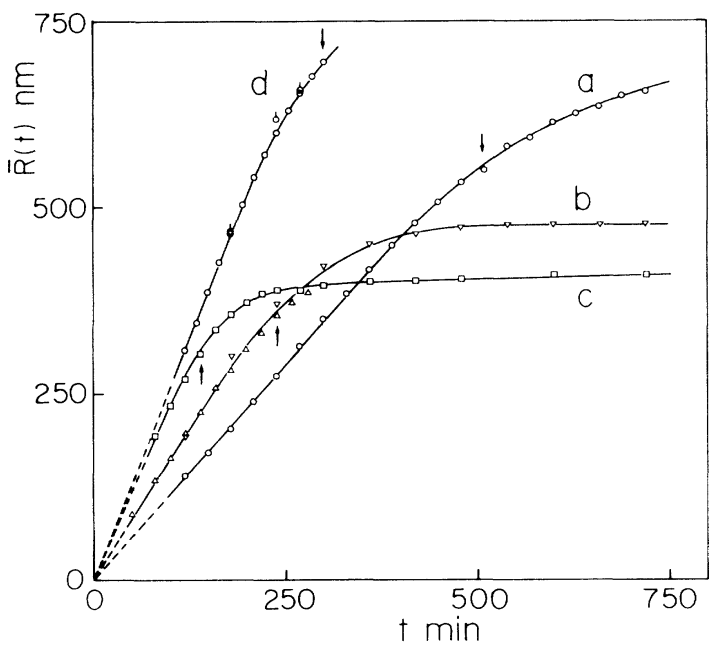

FIG. 3. Mean radius $\bar{R}(t)$ as a function of time $t$ after quench. Curves $a, b$, and $c$ were obtained for solution $B$ at quench depths $0.10,0.30$, and $0.50 \mathrm{~K}$, respectively, and curve $d$ for solution $E$ at $0.10 \mathrm{~K}$. Arrows indicate the time at which transmittance of solutions reduced to 0.90 . Points $\triangle$ and $\nabla$ for curve $b$, and $O$ and $O$ for curve $d$ are due to different experimental runs.

Figures 4 and 5 show the double logarithmic plots of $I(0, t)$ vs $t$ and $\bar{R}(t)$ vs $I(0, t)$, respectively, where the symbols are the same as in Fig. 3. Curves $b$ and $c$ in Figs. 3 and 4 show level-off behavior, which accompanied rapid slowdown in the decrease of transmittance $F$. The leveled-off systems became clear slowly from the top by the sedimentation of droplets indicating vanishing supersaturation.

In Fig. 6 the scattering curves are replotted in a scaled form with the reduced quantities $I(k, t) / I(0, t)$ and $\bar{R} k$. Data points $\bigcirc(0.10 \mathrm{~K}, 390 \mathrm{~min}, 0.98), \square(0.10 \mathrm{~K}, 690$ $\mathrm{min}, 0.82)$, and $\Delta(0.30 \mathrm{~K}, 240 \mathrm{~min}, 0.90)$ were obtained for solution $B$, and point $\nabla(0.10 \mathrm{~K}, 180 \mathrm{~min}, 1.00)$ was obtained for solution $E$. The values in parentheses indi-

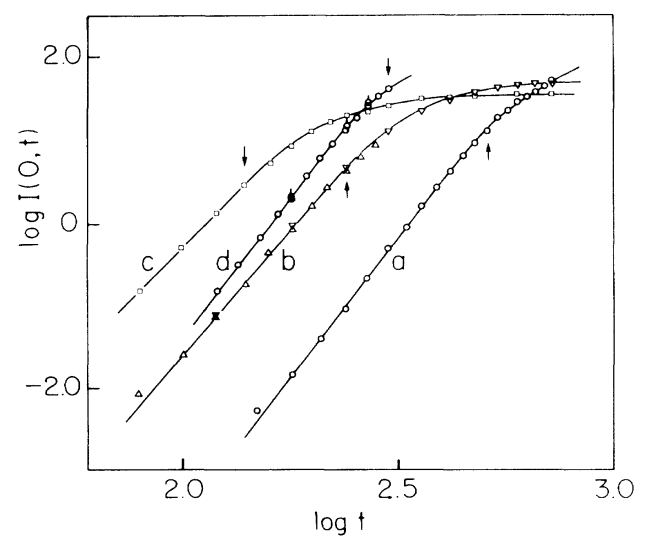

FIG. 4. Log-log plots of zero-angle scattering $I(0, t)$ vs time t. Symbols are the same as in Fig. 3.

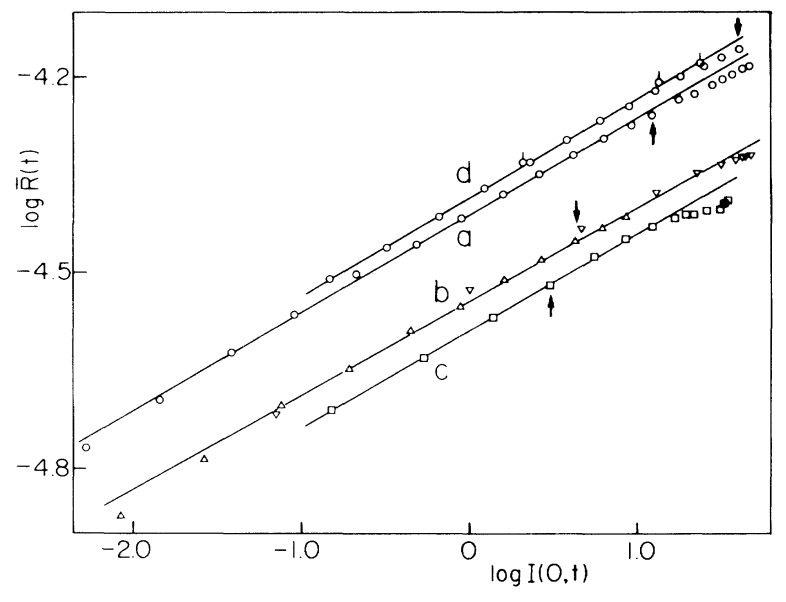

FIG. 5. Log-log plots of mean radius $\bar{R}(t)$ vs zero-angle scattering $I(0, t)$. Symbols are the same as in Fig. 3 .

cate $\delta T, t$, and $F$ from the left. Data points $O, \triangle$, and $\nabla$ show the same scattering behavior as described by the solid curve. Furthermore, the scaled scattering functions obtained for $F>0.90$ obeyed the same behavior as the solid curve irrespective of time, quench depth, and concentration. The different scattering behavior shown by data point $\square$ could be attributed to the effect of the multiple scattering, because the scattering functions obtained for $F<0.9$ exhibited similar deviation from the solid curve.

The unique behavior of the scaled scattering functions

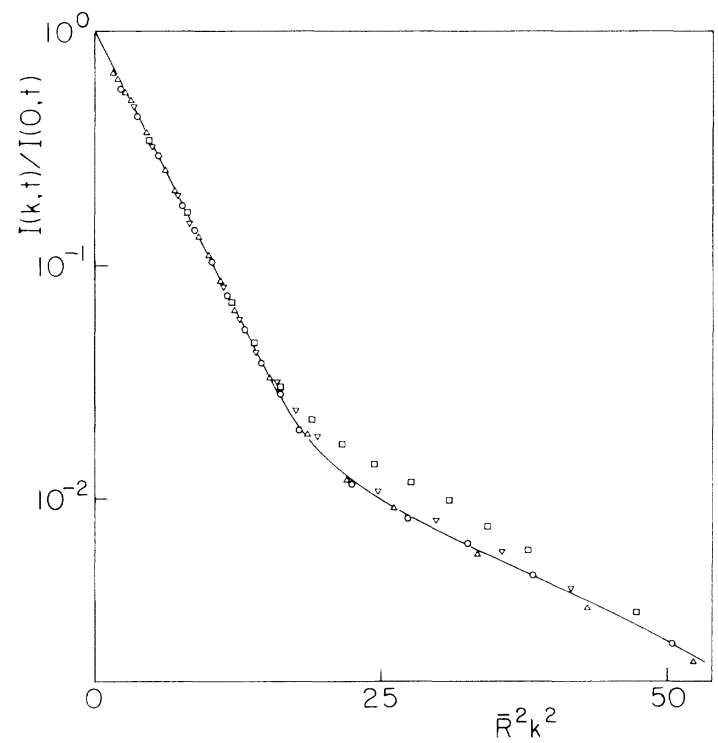

FIG. 6. Scaled scattering function by Guinier plot. Points $\triangle$, $O$, and $\square$ were obtained 240,390 , and $690 \mathrm{~min}$, after quench, respectively, for solution $B$, and $\nabla$ was obtained $180 \mathrm{~min}$ after quench for solution $E$. Quench depth is $0.30 \mathrm{~K}$ for point $\triangle$ and $0.10 \mathrm{~K}$ for the others. 
gives

$$
I(k, t)=I(0, t) P(x),
$$

where $P(x)$ is a function of $x \equiv \bar{R}(t) k$ independent of time. Substituting Eq. (4) into Eq. (2) and using $I(0, t)$ $=c \overline{\boldsymbol{R}}^{\gamma}$, the time evolution of the distribution function $N(R, t)$ can be written as

$$
\begin{aligned}
& N(R, t)=(\sqrt{\pi} / 2 A) c \bar{R}^{\gamma-7} H(R / \bar{R}), \\
& H(R / \bar{R})=(\bar{R} / R)^{3} \int_{0}^{\infty} x^{3} P(x) \Phi(R x / \bar{R}) d x .
\end{aligned}
$$

Equation (5) implies that $N(R, t)$ depends on time $t$ only through $\bar{R}(t)$. The total droplet density $N(t)$ and the volume fraction $v(t)$ of the minority phase are obtained from Eq. (5) by integration as $N(t) \sim c \bar{R}^{\gamma-6}(t)$ and $v(t) \sim c \bar{R}^{\gamma-3}(t)$. Assuming $\alpha=1$ and $\gamma=6.6$ at small $t$, we have $N(t) \sim c a^{0.6} t^{0.6}$, which indicates a droplet birth process in nucleation. With the normalized function $h(R / \bar{R})$ of $H(R / \bar{R})$, Eq. (5) is rewritten as $N(R, t)$ $=N(t) h(R / \bar{R}) / \bar{R}$, which has the same scaled form as the Lifshitz-Slyozov equation for a droplet growth process characterized by the relations $N(t) \sim t^{-1}$ and $\bar{R}(t) \sim t^{1 / 3}$ [15].

Langer and Schwartz [7] have derived rate equations for the phase-separation process near a critical point and predicted that $N(t)$ at a droplet birth process in nucleation is extremely sensitive to the degree of supercooling $\delta T / \Delta T_{c x}$ with $\Delta T_{c x}$ given in Fig. 1. For example, an increase of only 0.02 in $\delta T / \Delta T_{c x}$ would produce a $10^{2}$ - to $10^{3}$-fold increase in $N(t)$. In the present study the factor $c a^{0.6}$ shows the dependence of $N(t)$ on $\delta T / \Delta T_{c x}$, which ranged from 0.024 to 0.118 . For $\alpha=1$ and $\gamma=6.6, a, c$, and $c a^{0.6}$ were obtained as in Table $I$. Compared with the above prediction, the variation of $N(t)$ with $\delta T / \Delta T_{c x}$ is very small.

For supercooled solutions of polystyrene (PS) with $M_{w}=2.99 \times 10^{5}$ in cyclohexane near the critical point, a decrease in droplet density was observed a few minutes after the quench and attributed to the coalescence of droplets. In terms of the scaled time $\tau=t / t_{c}$ with $t_{c} \sim \xi^{2} / D[7]$, the present solutions far off the critical point might be expected to undergo faster phase separation than the PS solutions near the critical point. Here $D$ is the diffusion constant and $\xi$ is the correlation length proportional to the critical radius of the droplet, and therefore, to the interfacial tension $\sigma$. At present we cannot discuss the reason for the very slow phase separation, i.e., the large $t_{c}$ of the PMMA system, for lack of data on $\sigma$. However, it should be mentioned that the radius of the PMMA is comparable with the correlation length in the PS solutions.

In phase equilibrium experiments on dilute solutions of PMMA we could not observe a clear interface between two coexisting phases for the molecular weight $M_{w}$ $=2.44 \times 10^{6}$, but could for the smaller molecular weight $M_{w}=2.68 \times 10^{5}$. This observation suggests that interfacial tension measurements will be feasible for solutions of PMMA with low molecular weight. Therefore, phaseseparation experiments on the solutions could be compared with nucleation theories by using data on $\sigma$ to elucidate the very slow phase-separation process observed in this study.

We wish to thank $M$. Tanisaka for the construction of the light-scattering apparatus.

[1] B. E. Sundquist and R. A. Oriani, J. Chem. Phys. 36, 2604 (1962).

[2] R. B. Heady and J. W. Cahn, J. Chem. Phys. 58, 896 (1973).

[3] R. G. Howland, N. C. Wong, and C. M. Knobler, J. Chem. Phys. 73, 522 (1980).

[4] A. J. Schwartz, S. Krishnamurthy, and W. I. Goldburg, Phys. Rev. A 21, 1331 (1980).

[5] S. Krishnamurthy and W. I. Goldburg, Phys. Rev. A 22 , 2147 (1980).

[6] S. Krishnamurthy and R. Bansil, Phys. Rev. Lett. 50, 2010 (1983).

[7] J. S. Langer and A. J. Schwartz, Phys. Rev. A 21, 948 (1980).

[8] Y. C. Chou and W. I. Goldburg, Phys. Rev. A 23, 858 (1981).

[9] T. Hashimoto, M. Itakura, and H. Hasegawa, J. Chem. Phys. 85, 6118 (1986).

[10] M. Nakata and N. Numasawa, Macromolecules 18, 1736 (1985).

[11] M. Kurata, Thermodynamics of Polymer Solutions (Harwood, New York, 1982).

[12] Y. C. Chou and W. I. Goldburg, Phys. Rev. A 20, 2105 (1979).

[13] J. Riseman, Acta Crystallogr. 5, 193 (1952).

[14] J. Turkevich and H. H. Hubbell, J. Am. Chem. Soc. 73, 1 (1951).

[15] I. Lifshitz and V. Slyozov, J. Phys. Chem. Solids 19, 35 (1961). 